\title{
Contexto sociocultural familiar y nivel de desarrollo de las habilidades comunicativas en estudiantes de la Especialidad de Educación Primaria
}

\section{Sociocultural family context and level of development of communication skills in students of the Specialty of Primary Education.}

\author{
Marina Luz Navarro Guillén¹. Abelardo Rodolfo Campana Concha
}

\begin{abstract}
RESUMEN
Objetivo: Determinar el tipo de relación que existe entre la variable contexto sociocultural familiar y el nivel de desarrollo de las habilidades comunicativas de los estudiantes de educación superior no universitaria. Material y Métodos: Se empleó un diseño descriptivo correlacional, asimismo como instrumentos de recolección de datos se aplicó un cuestionario y un test que fueron aplicados a una muestra de 170 estudiantes para recolectar información sobre las variables en estudio, cuyas hipótesis emplearon el coeficiente de correlación de Pearson para establecer el nivel de relación. Resultados: Existe una relación positiva muy alta entre el contexto social familiar y el nivel de desarrollo de habilidades para escuchar y hablar de los estudiantes. Conclusión: Existe una relación positiva muy alta entre el contexto cultural familiar y el nivel de desarrollo de habilidades para elaborar textos de los estudiantes; los cuales se han evidenciado en una correlación de $r=0,9510$ en la primera hipótesis específica y un $r=0,9782$ en la segunda hipótesis específica.
\end{abstract}

Palabras clave: Contexto sociocultural familiar, relación positiva, contexto social y cultural familiar, habilidades comunicativas de los estudiantes.

\section{ABSTRACT}

Objective: To determine the type of relationship that exists between the family sociocultural context variable and the level of development of the communication skills of non-university higher education students. Material and Methods: A descriptive correlational design was used, as well as data collection instruments, a questionnaire and a test were applied that were applied to a sample of 170 students to collect information on the variables under study, whose hypotheses used the correlation coefficient. of Pearson to establish the level of relationship. Results: There is a very high positive relationship between the family social context and the level of development of listening and speaking skills of the students. Conclusion: There is a very high positive relationship between the family cultural context and the level of skills development to elaborate student texts; which have been evidenced in a correlation of $r=0,9510$ in the first specific hypothesis and a $r=0,9782$ in the second specific hypothesis.

Keywords: Family sociocultural context, positive relationship, family social and cultural context, student communication skills

\section{INTRODUCCIÓN}

Desde hace algunos años atrás, la educación y más concretamente la educación superior no universitaria se encuentra en proceso de acreditación. Como parte importante del diagnóstico para lograr la acreditación, resalta como uno de los principales problemas la baja calidad de los aprendizajes de los estudiantes. Dentro de esta baja calidad de los deficiente nivel de desarrollo alcanzado en las habilidades comunicativas y comprensivas de los estudiantes. Por otra parte, los factores asociados a esta deficiencia, son múltiples, pero uno de esos factores que generalmente no se ha tomado en cuenta es las condiciones socio culturales de los miembros de la familia, que no solamente tienen efecto en los niños en edad escolar, sino también en los estudiantes de educación superior.

Es precisamente esta situación lo que motiva a definir el problema de investigación de tipo correlacional, "Relación entre el contexto sociocultural familiar y nivel de desarrollo de las habilidades comunicativas en estudiantes del IST Público "Catalina Buendía de Pecho" de Ica".

Cuando no se tiene un nivel adecuado de desarrollo de las habilidades comunicativas como es la comprensión, expresión y producción de textos, no hay forma de lograr un aprendizaje, así como la limitación de nuestro desarrollo personal, debido a que no mantienen un acercamiento con los textos disponibles en nuestro entorno. Es por ello que consideramos relevante mejorar los hábitos de lectura, la expresión y la producción de textos que son habilidades muy importantes para el estudiante de educación superior.

\section{MATERIAL Y MÉTODO}

Es una investigación cuantitativa de nivel explicativo ex

${ }^{1}$ Estudiante Maestría: Docencia Universitaria Facultad De Educación, UNMSM aprendizajes, se encuentra como uno de los indicadores el

posfacto, con el cual se busca identificar el nivel de relación presente en las variables de estudio, por ello también es catalogado como una investigación es no experimental de diseño correlacional.

Asimismo, se empleó la técnica del Azar simple para la selección de la muestra

\section{RESULTADOS}

Para obtener los resultados, se tuvo que realizar un análisis estadístico, para ello se empleó la prueba de hipótesis es con el coeficiente de correlación de Pearson para datos agrupados.

\section{Estos se manifiestan de la siguiente manera:}

contrastación de la primera hipótesis específica

Ha. Entre el contexto social familiar y las habilidades para escuchar y hablar de los estudiantes existe una relación positiva.

Tabla 1.

Hipótesis específica

\begin{tabular}{lcccccc}
\hline $\begin{array}{l}\text { variable } \\
\quad \text { Y1 }\end{array}$ & MA & II & III & IV & V & \\
variable & $42-50$ & $34-41$ & $26-33$ & $18-25$ & $10-17$ & $\mathrm{~N}_{\mathrm{x}}$ \\
$\quad \mathrm{X} 1$ & & & & & & \\
\hline $\begin{array}{l}\text { Muy alto } \\
42-50\end{array}$ & 28 & & & & & 28 \\
$\begin{array}{l}\text { Alto } \\
34-41\end{array}$ & 2 & 40 & 2 & & & 44 \\
$\begin{array}{l}\text { Regular } \\
26-33\end{array}$ & & 2 & 46 & 10 & & 58 \\
$\begin{array}{l}\text { Bajo } \\
18-25\end{array}$ & & & 2 & 18 & 4 & 24 \\
$\begin{array}{l}\text { Muy } \\
\text { Bajo }\end{array}$ & & & & 2 & 14 & 16 \\
$10-17$ & & & & & & \\
\hline $\begin{array}{l}\mathrm{N}_{\mathrm{y}} \\
\text { Fuente: Navarro (2018) }\end{array}$ & & & & & & \\
\hline
\end{tabular}

Big Bang Faustiniano revistas.unjfsc.edu.pe: 
Habiéndose establecido un nivel de correlación de $\mathrm{R}=\mathrm{el}$ coeficiente de correlación de $r=0.9510$ se demuestra existe una muy alta correlación entre las variables $\mathrm{X} 1$ e $\mathrm{Y} 1$ por cuanto este valor está relativamente cercano a +1 . Asimismo, se rechaza la hipótesis nula.

\section{contrastación de la segunda hipótesis específica}

Ha. Entre el contexto cultural familiar y las habilidades para elaborar textos de los estudiantes existe una relación positiva

Tabla 2.

Hipótesis específica 2

\begin{tabular}{lcccccc}
\hline \multicolumn{1}{c}{ variable } & I & II & III & IV & V & \\
$\quad$ Y1 & MA & A & R & B & MB & $\mathrm{N}_{\mathrm{x}}$ \\
variable & $18-20$ & $15-17$ & $12-14$ & $09-11$ & $06-08$ & \\
$\quad$ X1 & & & & & & \\
\hline $\begin{array}{l}\text { Muy alto } \\
42-50\end{array}$ & 26 & 4 & & & & 30 \\
$\begin{array}{l}\text { Alto } \\
34-41\end{array}$ & 2 & 40 & & & & 42 \\
$\begin{array}{l}\text { Regular } \\
26-33\end{array}$ & & 2 & 46 & 4 & & 52 \\
$\begin{array}{l}\text { Bajo } \\
18-25\end{array}$ & & & & 22 & 6 & 28 \\
$\begin{array}{l}\text { Muy } \\
\text { Bajo } \\
10-17\end{array}$ & & & & 4 & 14 & 18 \\
\hline $\begin{array}{l}\mathrm{N}_{\mathrm{y}} \\
\text { Fuente: Navarro(2018) }\end{array}$ & & & & & & \\
\hline
\end{tabular}

Se determinó un nivel de correlación de $r=0.9584$ se demuestra una muy alta correlación entre las variables $\mathrm{X} 2$ y $\mathrm{Y} 2$. Confirmándose una asociación significativa.

\section{Prueba de hipótesis general}

Ha. El aspecto sociocultural familiar y las habilidades comunicativas de los estudiantes del Instituto Superior Tecnológico Público "Catalina Buendía de Pecho de Ica, existe relación positiva.

Habiéndose validado las hipótesis específicas 1, y 2, la hipótesis general también queda validada. Esta validación es consistente lógicamente por cuanto mediante el proceso de operacionalización de las variables generales de la hipótesis general X y, se ha transformado en dimensiones o indicadores, con los que a su vez se ha construido las hipótesis específicas 1 y 2; es por ello que al validarse estas hipótesis específicas, también se validad la hipótesis general.

Pero, además, como se ha observado en los diferentes cuadros estadísticos se demuestran que existe una relación directa positiva tanto en la primera como en la segunda hipótesis específica, lo que prueba la hipótesis general.

Además de estos datos, existe un fundamento teórico que sustenta a la hipótesis general. De acuerdo con los conocimientos sistematizados en el marco teórico, la educación y en general el aprendizaje del idioma no se inicia en la escuela, sino en la casa. La casa y la familia es la primera escuela para el niño y sus aprendizajes para lo posterior.

Particularmente el aprendizaje del idioma, del lenguaje oral y escrito, es una habilidad adquirida y por lo mismo, los primeros años de formación en la casa son claves para el aprendizaje posterior de la forma cómo una persona se expresa o elabora textos. Naturalmente la escuela lo modifica y lo perfecciona, sin embargo, las bases para el aprendizaje se construyen en la familia, en las relaciones familiares; es decir en el contexto socio familiar.

Las funciones intrapersonales (el lenguaje, desarrollo simbólico, solución de problemas, interiorización de conceptos, atención, memoria) se origina en un contexto interpersonal lo cual según las teorías de Vigotsky, el desarrollo del niño, sus funciones aparece en dos ocasiones: tanto en un ámbito social e individual; se inicia entre las personas, como en el propio niño. Esta doble aparición es denominado como zona de desarrollo próximo que consiste en que la situación es producida mediante la interacción, asimismo lo considera necesario para el desarrollo del lenguaje del niño, fundamental para que se vaya desarrollando en su vida adulta, en otras palabras, la interacción con su entorno ayudará al fortalecimiento de sus destrezas, habilidades.

\section{DISCUSIÓN}

La investigación sostiene básicamente la existencia de una relación positiva entre el entorno sociocultural familiar y las habilidades comunicativas, de modo las muestras proceden de ambiente sociocultural adecuado o positivo, tienen desarrollado de las habilidades comunicativas, mayor a los que provienen de ambientes socioculturales adversos.

Guzmán y Pacheco (2014) en su investigación sobre Comunicación familiar y desempeño académico en estudiantes universitarios de Colombia, reporta evidencias empíricas que los estudiantes de condición social bajo generalmente fracasan o abandonan el estudio.

Parra (2013) por su parte, al investigar sobre el pensamiento crítico y habilidades comunicativas en estudiantes universitarios chilenos, también hace conocer que los estudiantes que tienen mejores habilidades comunicativas, proceden de contextos socioculturales familiares positivos.

En el ámbito nacional, Bustamante (2014) plantea y reporta que el programa de habilidades comunicativas mejora el nivel de comprensión lectora de los alumnos de nivel secundaria, ya que en la prueba inicial y prueba final se evidenciaron mejoras en sus calificaciones en la mayoría de la población.

Gonzáles (2006) y Guzmán (2007) de la Universidad Nacional de Trujillo, tienen similares conclusiones al afirmar que el desarrollo de las habilidades comunicativas se ve afectada también por el contexto familiar de los estudiantes.

Como hemos evidenciado que son diversos investigadores evidenciaron que el nivel socioeconómico incide en el rendimiento académico, incluso perjudicando a la finalización de la educación básica, podemos mencionar a González (2003) admite ello, pero no aclara específicamente hasta qué punto determina los resultados de la práctica pedagógica, ya que también existen casos que las limitaciones sociales y económicas no determinaron su desarrollo académico.

Guevara, Jaramillo y Tovar (2013), mencionando a Urquijo (2005), también aceptan el planteamiento anteriormente mencionado, sin embargo en contrastación con lo planteado anteriormente, conciben el promedio académico, las expectativas frente al desarrollo profesional en el nivel superior no guardan relación significativa con el factor socioeconómico, el tener condiciones desfavorables socioeconómicas implica la presencia de problemas en su aspecto formativo y a su vez perjudicaría la culminación exitosa de la formación profesional, contrastando con aquellos, asimismo considera que los aspectos socio económicos y culturales, en las escuelas públicas y privadas y la estimulación familiar tienen relativa incidencia en sus calificaciones.

Guevara, Jaramillo y Tovar (2013) también establece que el desarrollo escolar cobra importancia y necesidad para la formación del estudiante para su futuro que puede ser reflejado en el desarrollo personal y la satisfacción de necesidades de superación, de adquisición de conocimiento y de desarrollo de capacidades personales para proyectarse metas y objetivos, 
asimismo analizando los logros alcanzados, como una forma de alcanzar logros y metas, por ello si uno se establece bien claramente estos puntos los estratos económicos no cobrarían mayor relevancia.

Por su parte, Papalia (1999) considera que el desarrollo intelectual sucede precisamente entre la etapa de la adolescencia a la adulta temprana, la cual está en relación al conocimiento adquirido por las personas las cuales representan lo que es importante y significativo en sus vidas, ya que su desempeño tendrá mayor repercusión en aquellas tareas que tengan importancia para las metas que se han fijado en la vida.

Siguiendo esas líneas de análisis sobre el nivel educativo y el entorno social - económico, existen investigaciones en las que señalan que la educación básica existe un fuerte vínculo con dichos aspectos, sin embargo, en la etapa universitaria ya no es tan notorio o necesario, puesto que las familias se encuentran en una etapa del ciclo vital en la que los hijos manejan un mayor nivel de independencia y de responsabilidad de su quehacer y de sus aspiraciones, similares puntos de vista son presentados por Castejón (1996) quien considera que el nivel cultural de los padres, expresado en lo académico, influyen en el rendimiento académico de los estudiantes, ya que en esta etapa del desarrollo los niños dependen de sus padres para su desarrollo cognitivo, puesto que representan a los principales orientadores y acompañantes permanentes, razón por la cual la formación académica de los mismos es necesario e importante.

En similitud con lo señalado anteriormente, Morales et al. (1999) resumen que el nivel cultural familiar tiene una estrecha relación con el rendimiento escolar de los niños; es por eso que cuando el nivel de formación de los padres está determinado por la no culminación del nivel básico de sus estudios o el analfabetismo, existe mayor posibilidad a que los hijos no puedan llevar a buen ritmo sus estudios, probablemente porque no podrá a reforzar en casa los conocimientos adquiridos en el aula; a diferencia de aquellos padres con un nivel de formación medio o alto es más probable encontrar un rendimiento bueno.

\section{CONCLUSIONES}

El desarrollo del lenguaje depende de la influencia que tenga la familia y la escuela, puesto que el primero al ser el entorno más allegado al niño para su desarrollo comunicativo y socializador es necesario enriquecerlos de buenas experiencias para que pueda mejorar los aspectos comunicativos.

La mayoría de los estudiantes del Instituto de educación superior Pedagógico Público "Catalina Buendía de Pecho" de Ica, tiene niveles de desarrollo de habilidades comunicativas asociadas a las condiciones socioculturales familiares en los que se desenvuelven.

Existen diversos factores asociados al progreso con respecto a las habilidades comunicativas de los estudiantes de la población donde se desarrolló el estudio; entre estos factores se sobresalen las condiciones sociales y culturales familiares.

Entre las habilidades para escuchar y hablar, y el tipo de comunicación intrafamiliar de los estudiantes de la institución en análisis, existe una relación positiva; de modo que, a mayor habilidad existe mayor posibilidad de mejora en cuanto a las condiciones de comunicación intrafamiliar, lo cual se evidencia en la correlación de Pearson que resultó $r=0.7635$.

Entre el tipo de relaciones sociales entre los miembros de la familia y desarrollo de las habilidades para elaborar textos de los estudiantes del Instituto Superior Tecnológico Público "Catalina Buendía de Pecho de Ica, existe una relación positiva, lo que se demuestra mediante la correlación de Pearson que identificado con el $r=0.9578$.

\section{RECOMENDACIONES}

La Dirección Instituto de Educación Superior Pedagógico Público "Catalina Buendía de Pecho" de Ica, debe implementar acciones pedagógicas para fortalecer en los estudiantes el desarrollo de habilidades comunicativas, como parte importante del proceso de acreditación institucional.

Los profesores del Instituto de Educación Superior Pedagógico Público "Catalina Buendía de Pecho" de Ica, deben implementar estrategias didácticas activas y motivadoras para fortalecer el logro de habilidades para la expresión y producción de textos de los estudiantes, a fin de mejorar la formación profesional de éstos.

Se debe llevar a cabo cursos y seminarios taller para mejorar la expresión oral de los estudiantes del Instituto de Educación Superior Pedagógico Público "Catalina Buendía de Pecho" de Ica.

Se debe llevar a cabo cursos y seminarios taller para mejorar la producción de textos de los estudiantes del Instituto de Educación Superior Pedagógico Público "Catalina Buendía de Pecho" de lca.

\section{REFERENCIAS BIBLIOGRÁFICAS}

Bustamante, M. (2014). Programa de habilidades comunicativas para mejorar el nivel de comprensión lectora en los alumnos de $5^{\circ}$ de secundaria del centro educativo particular "Juan Pablo II"- Trujillo- Perú (Tesis Posgrado) Universidad Antenor Orrego, Trujillo, Perú.

Castejón, J. (1996). Determinantes del rendimiento académico de los estudiantes y de los centros educativos: modelos y factores. España: Editorial Club Universitario.

Gonzáles, P. (2006). Influencia del proceso de la decodificación lectora en el aprendizaje de la comprensión lectora de los alumnos del 3er. grado en la escuela de San Isidro. Distrito de Chugay provincia de Sánchez Carrión. La Libertad (Tesis Posgrado) Universidad Nacional de Trujillo, Trujillo, Perú.

González, C. (2003). Factores determinantes del bajo rendimiento académico en educación secundaria (Tesis Posgrado) Universidad Complutense de Madrid, Madrid, España. Recuperado en: https://eprints.ucm.es/4802/.

Guevara, E.; Jaramillo, R. y Tovar, S. (2013). Factores familiares y su relación con el rendimiento académico en estudiantes de psicología. Revista Virtual Universidad Católica del Norte, 40 (2013). Recuperado en: http://www.redalyc.org/pdf/1942/1942292000 09.pdf

Guzmán, D. (2007) Programa para desarrollar la habilidad de la lectura y su influencia en el nivel de comprensión lectora de los estudiantes de en la Institución Educativa La Salle de la ciudad de Trujillo (Tesis Posgrado) Universidad Nacional de Trujillo, Trujillo, Perú.

Guzmán, R. y Pacheco, M. (2014). Comunicación familiar y desempeño académico en estudiantes universitarios. Zona Próxima, 20 (2014), pp. 79-91. Recuperado en: http://www.redalyc.org/pdf/853/85331022008.pdf

Morales, A. et al. (1999). El entorno familiar y el rendimiento escolar. España: Proyecto de Investigación Educativa subvencionado por la Consejería de Educación y Ciencia de la Junta de Andalucía. Recuperado en: http://cania.msinfo.info/bases/biblo/texto/pdf2 entorno familia.pdf 
Papalia, D. (1999). Desarrollo Humano. Bogotá - Colombia: Mc Graw Hill

Parra, I (2013). Desarrollo de habilidades del pensamiento crítico y su incidencia en la fluidez verbal en los estudiantes de la facultad de filosofía de la universidad de Guayaquil, propuesta: guía de estrategias (Tesis Posgrado) Universidad de Guayaquil, Ecuador. $R$ e c u p e r a d o e $n$ : http://repositorio.ug.edu.ec/bitstream/redug/2035/1/tes is\%20maestria\%20definitiva\%20ultima.pdf 